\title{
THE SCIENTIFIC PROJECT OF THE SMALL SATELLITE OF CHINA $\dagger$
}

\author{
WEN-RUI Hu \\ Institute of Mechanics, Chinese Academy of Sciences, 15 Zhong Guan Cun Road, Beijing 100080, \\ People's Republic of China \\ and \\ HENG Du \\ Center for Space Science and Application Research, Chinese Academy of Sciences, Beijing 100080, \\ People's Republic of China
}

\begin{abstract}
The efforts involved in developing a small satellite for scientific purposes in China in recent years are introduced in the present paper. The project is arranged on a case to case principle depending upon requirements and financial support. The space technology of a satellite and rockets, which have been developed over a relatively longer period in China, have been transferred to the scientific research of small satellites for improvement of the quality requirements. The surplus payloads of the rocket and satellite are used as the payloads of the small satellite and scientific experiments at a low cost. As an example, the project of balloon satellites for atmospheric research was successfully completed in 1991. The experience of the project management is of great benefit for further organization and arrangement of other projects. Opportunities exist for surplus payloads to be used in the future, and a small satellite for magnetospheric research will be launched in 1993.
\end{abstract}

\section{INTRODUCTION}

Space activities are mainly national activities, and are contributed by scientists and technicians from institutes all over China. The Chinese Government made the important decision to develop space technology in the late 1950s, and to organize the ministries for aerospace industry and institutions to perform missions with rockets, satellites and other space technologies. However, space science programs and applications in China are relatively disadvantaged in comparison to space technology. However, the development of space technologies, such as the rocket and satellite, support the basis for the development of space science, especially the scientific satellite.

In principle, the Chinese Academy of Sciences (CAS) is actively undertaking to solve national issues in science and technology, and is assigned to be the leading agency for such projects. It is giving full attention to its distinguished features of being a multi disciplinary and comprehensive research organization, and CAS has conducted extensive research in the field of natural science. Some fields of space science, such as space physics and space life science, have been evident in China since 1960s, and these fields have been extended to astronomy, earth

$\dagger$ Invited paper 1-M.3.01 presented at the 43 rd Astronautical Congress, Washington, D.C., U.S.A., 28 August5 September 1992. science, microgravity science and others in the last few years. Many scientific space experiments have been completed on board the Chinese satellite, and several scientific satellites have been arranged in recent years [1].

The Chinese scientific satellite project was started in the late 1960s, and was named the SJ (Shi-Jian, which means practice) system [2]. The Chinese scientific satellites are summarized in Table 1.

There are some changes in the organization of the institutes for scientific satellite business. The scientific designs of SJ-1 and SJ-2 were contributed by the Institute of Space Physics, which was founded in the Ministry of Aerospace Industry (MAS) in the late 1960s and returned to CAS in the late 1970s. The first three SJ satellites were completely in the charge of MAS. The astromony satellite was the first attempt to design and manufacture scientific satellite, from both satellite technical and scientific aspects, by CAS in late 1979. Unfortunately, the program was delayed indefinitly in 1985 due to adjustment of the state economy. Recently, the scientific satellite project has been rearranged after a ten year interruption, and will be continued under the care of the Chinese Academy of Sciences collaborating with MAS for space technology. According to the current situation in China, it seems that the scientific satellite program will mostly be of a small or medium size in the near future.

Many Chinese scientists have appealed for the development of space science and the scientific 
Table 1. List of Chinese scientific satellites

\begin{tabular}{llcl}
\hline Name & Launch time & Weight (kg) & Purpose \\
\hline SJ-1 (SKW-2) & 3 March, 1971 & 221 & Space physics \\
SJ-2 (SKW-3) & 20 Sept., 1981 & 257 & Space physics \\
SJ-3 & Cancelled in 1985 & 450 & Earth sciences \\
Astronomy satellite & Cancelled in 1985 & 500 & Astronomy \\
DQ-1A & 3 Sept., 1990 & 2.6 & Upper atmosphere density \\
DQ-1B & 3 Sept., 1990 & 3.3 & Upper atmosphere density \\
SJ-4 & 8 Feb., 1994 & & Magnetosphere \\
\hline
\end{tabular}

satellite, and hope that space science will be arranged to co-ordinate with space technology. At the present time, the program of the Chinese scientific satellite can be arranged based on a case by case principle, that is, it depends upon the scientific requirement, budget, opportunity for space technical support, particularly the launch opportunity. Generally, the requirement of the scientific satellite is suggested by the scientists of CAS, and the proposal will be put forward to the state authority on behalf of a principle institute, for example, the Center of Space Science and Application Research, collaborating with the institutes of MAS. A joint team led by a principle investigator(s) will be chosen to take care of the scientific satellite project after the application is accepted. The collaboration between CAS and MAS has been going smoothly over the last few years.

The first project concerning the small scientific satellite in China was the two balloon satellites, DQ-1A and DQ-1B, for upper atmospheric research during 1989-1991. The experience of the project management is of great help for further research. The second project was a scientific satellite which was launched in 1994 for magnetospheric research. Several proposals for scientific satellites have been suggested, and are expected to be arranged step by step. We will discuss the projects of DQ-1 and SJ-4 in the next se ions, respectively, and then the prospects and conclusions.

\section{PROJECT DQ-1 OF THE BALLOON SATELLITES}

The scientific purpose of a balloon satellite is to study density variation in the upper atmosphere, especially during the period of solar maximum, and the influence of solar and geomagnetic activities on the variation of upper atmospheric density. The density of the upper atmosphere is an important quantity for atmospheric physics, and has also important meaning on the application of near-earth environment. From a physics view point, many subjects need density data, such as the relation between the solar activities and the atmospheric response, the coupling between the atmosphere and the ionosphere and the coupling between the upper and middle atmosphere. It is of more interest to study the relation and coupling in the solar maximum period, when the solar and geomagnetic activities are stronger and more frequent. From the view point of application, most artificial satellites and space infrastructures have been operated in near-earth space, and the density of the environment is sensitive to the orbits and safety of the satellite and infrastructure. The larger the spacecraft, the larger the influence. The orbit density may be demonstrated by the falling trajectory of a spherical balloon satellite, and this is an effective and low-cost approach for obtaining density data.

The project DQ-1 ( $D \mathrm{a}-Q \mathrm{i}$, the Chinese name for atmosphere) involves two balloon satellites $A$ and $B$, that is, the DQ-1A and DQ-1B. The proposal was submitted jointly by several institutes in March of 1988 to the National High Technology Development Program, and later accepted. The principle institute was the Center for Space Science and Application Research, CAS. Phase A and phase B of the project worked until October of 1988 , and then phase $C$ and D till July of 1990. The satellites were launched together with the Chinese Meteorological Satellite FY-1 on 3 September 1990, and the orbit inclination was $99^{\circ}$ with a nominal orbit of $900 \mathrm{~km}$ in height. Two balloons perished, respectively, on 14 March 1991 and 23 July 1991. The satellite project worked fluently according to the schedule shown in Table 2. Numerous density data were obtained, and the analyses and studies have been continuous until now.

The upper atmospheric density above $500 \mathrm{~km}$ is not easy to measure directly by methods such as the mass spectrometer and ionization gauge, on the other hand, this region is above the best measuring distance of the incoherent scatter radar. The measurement principle of the balloon satellite is based on the momentum equilibrium,

$$
m \frac{\mathrm{d} v}{\mathrm{~d} t}=\frac{1}{2} C_{\mathrm{a}} \rho v^{2} A,
$$

where $m, A, v$ and $C_{a}$ are, respectively, the mass, cross section, velocity and drag coefficient of the balloon satellite and $\rho$ is the local density of the atmosphere. The density can be obtained if the velocity and acceleration of the satellite are observed by the ground station and $C_{\mathrm{a}}$ and $A$ are measured in the laboratory on the ground. In principle, the trajectory of one balloon satellite is enough to demonstrate the density, however, two satellites with different mass to cross section ratios give two independent data

Table 2. Phase schedule of project DQ-1

\begin{tabular}{|c|c|c|c|c|c|c|}
\hline \multirow{3}{*}{$\begin{array}{l}\text { DQ-1A } \\
\text { DQ-1B }\end{array}$} & \multicolumn{2}{|c|}{1988} & 1989 & 1990 & 1991 & 1992 \\
\hline & A & B & \multicolumn{2}{|c|}{$\mathrm{C} / \mathrm{D}$} & In orbit & Analyses \\
\hline & A & B & \multicolumn{2}{|c|}{$\mathrm{C} / \mathrm{D}$} & In orbit & Analyses \\
\hline
\end{tabular}


systems, useful for double checking against each other. The results show that the correlation coefficient between the two data systems is 0.9935 , as given in Fig. 1. This method is relatively simple in technology, is of low cost and easy to manage, and this is especially important for the first small mission of the scientific satellite.

The joint team of the project consists of four institutes and a network of ground stations of institutes from CAS and MAS (as shown in the following). The collaboration between the institutes is successful and effective.

(1) Center for Space Science and Application Research, CAS (principle institute)

- In charge of the overall project

- Designs the scientific purpose of the project

- Manufactures the balloon satellite

- Performs research on atmospheric physics.

(2) Shanghai Bureau, MAS

- Rocket utilization of CZ-4

- Installs the balloon satellites for launch

- Develops the ejector for separating the satellite from the rocket.

(3) Chinese Academy of Space Technology (CAST), MAS

- In charge of the overall technology

- Performs stimulation experiments on the space environment

- Studies atmospheric density.

(4) Center of Artificial Satellite Application, CAS

- Observation and calculation of the satellite trajectories

- Demonstrates atmospheric density from the observation data.

- Studies the model of the upper atmosphere.

(5) Department of Geophysics, Peking University

- Studies upper atmospheric physics.

The collaborating institutes consist mainly of two parts, one part is the CAS institutes and university, which take care of the scientific fields and hardware

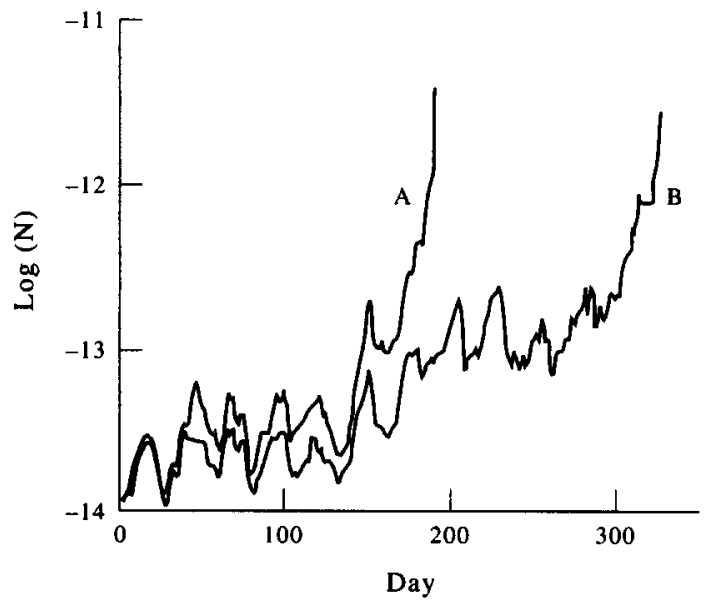

Fig. 1. The correlation between the two data systems, DQ- $1 \mathrm{~A}$ and DQ-1B development of the project; the other part is the MAS institutes, which contribute mainly to the space technology, such as the rocket and satellite technology, of the project. The experts of MAS have worked on the development of space technology for a long time, their ripe experience is important to guarantee the reliability of the scientific satellite. The collaboration also supports the opportunity to use surplus payload weight for launching a scientific mission and decreases the cost.

The DQ-1A and DQ-1B satellites successfully operate in orbit. All the engineering properties closely satisfied the requirements of the design. The scientific payload was ejected into the predicted circle orbit as shown in Table 3.

The payload expands into the two spherical balloons due to residual gas and sublimation of the materials in the folding balloons. The balloon satellite keeps the form of a sphere during its lifespan, however, there are different satellite lifes due to the different ratios of mass to cross section of the satellite; one satellite life is nearly half a year while the other is nearly one year. The materials of the DQ-1 satellites are of high quality, and also possess a high brightness for ease of observation from the ground. The satellites were quickly observed by the Chinese observation network after the payload expanded into the spherical balloons in orbit.

Many data were obtained by the Center of Artificial Satellite Application, which consists of seven ground stations at astronomical observatories. The stations are located in Nanjing (E113, N32), Beijing (E116, N40), Xi-an (E109, N34), Kunming (E102, N25), Changchun (E125, N44), Guangzhou (E113, $\mathrm{N} 23$ ) and Urumuqi (E88, N44). The headquarters of the center is located in the Purple Mountain Astronomical Observatory of Nanjing. Figure 2 gives a set of typical data of DQ-1, which agree with those obtained abroad in order of magnitude and show the influence of solar activities and the solar rotation period of 27 days. It should be noted that solar activity is at a relatively high level in some periods of the satellite life, for example, in June of 1991, the balloon satellite was falling quickly during this period due to the increase of atmospheric density. The details of the density variation data are useful for studying the solar-terrestrial relation.

The experience of the DQ-1 project may be surmmarized as follows, and is useful for further small scientific satellite arrangements.

(1) The small scientific satellite can be arranged on a case by case principle, at the present time

Table 3. The orbit parameters of the DQ-1 payload

\begin{tabular}{lcc}
\hline & Design value & Real value \\
\hline Apogee $(\mathrm{km})$ & 7273.773 & 7276.711 \\
Perigee $(\mathrm{km})$ & 7272.391 & 7271.616 \\
Eccentricity & 0.000095 & 0.00037 \\
Inclination $(\cdot)$ & 99.900 & 98.9585 \\
Period $(\mathrm{s})$ & 6172.892 & 6174.270 \\
\hline
\end{tabular}




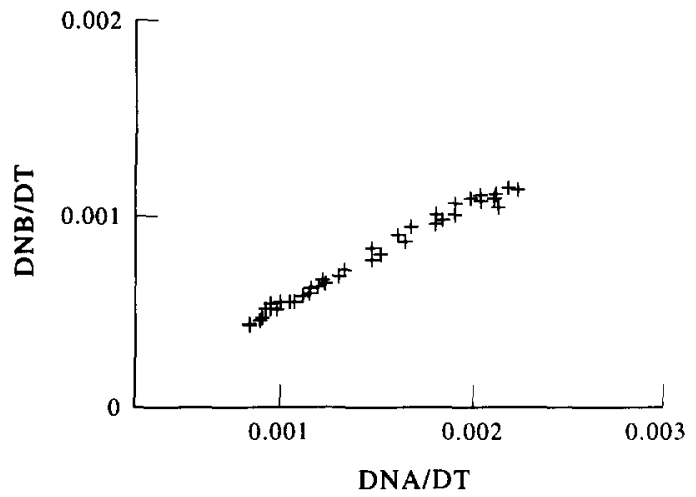

Fig. 2. Typical results of the density variation obtained by balloon satellite DQ-1.

based on mission requirement and opportunity in China.

(2) The inter-agency collaboration between the scientists of CAS, who are in charge of the scientific aspect and hardware development of the space experiment, and the experts of MAS, who take care of the space technology, is of benefit to the performance of the small satellite mission.

(3) It is a low-cost approach to launch the scientific satellite together with another main mission. In this case, the surplus payload weight of the rocket is used for the small scientific satellite.

(4) Transferring the developed technology of satellite and rocket engineering to the scientific satellite is an effective and low-cost approach.

After the first mission of the Chinese scientific satellite, we hope there will be more missions in the future.

\section{PROJECT OF THE SCIENTIFIC SATELLITE SJ-4}

Solar-terrestrial science is one of the front fields in space science[3]. There are several programs organized in China for co-ordinated research in this field, for example, the program on the Global Character of Solar-Terrestrial System in Antarctic Research (1992-1996) supported jointly by CAS and the Office of National Antarctic Research, and the program on Solar-Terrestrial Energy Transportation (1992-1997) of the National Science Foundation. However, these programs involve no direct measurement of the Chinese scientific satellite. Several dozens of satellites will be launched in the 1990s by the Solar-Terrestrial Science Project of the Inter-Agency Consultative Group (IAGG) for solar-terrestrial research. It is also proposed to launch the Chinese scientific satellite, which will contribute to the international community of space physical research. The project of the scientific satellite $\mathrm{SJ}-4$ has been accepted as a national space program.

The scientific purpose of satellite SJ-4 is to study the influence of solar and magnetospheric activities on the distribution of plasma and energetic particles in earth space from near-earth orbit to near-earth stationary orbit, which covers the regions of the ionosphere, upper atmosphere, plasma sphere, inner and outer radiation belts and the region of trapping energetic particles. The measurements of plasma and particle distributions may shed light on many ionospheric and magnetospheric processes, such as the transportation of mass and energy, the mechanism of magnetospheric storm and substorm, the coupling between different typical regions, the distribution and origin of oxygen ions in the magnetosphere and the response of particle and plasma states to solar and magnetospheric activities. On the other hand, the orbit of SJ-4 covers the important regions of satellite environments, and the data will also be of benefit to satellite design and operation.

SJ-4 is a small satellite, but has all the functions of a larger satellite and the same procedure is followed from design, manufacture, integration, test, launch and operation. The weight of the scientific payloads is less than $20 \mathrm{~kg}$. The program will be jointly completed by the two following institutions.

(1) Center for Space Science and Application Research, CAS

- Designs the scientific purpose and requirements of SJ-4

- Manufactures and integrates scientific payloads

- Receives data from the orbiting satellite

- Analyzes the data and carries out research on space physics.

(2) Ministry of Aerospace Industry

- Designs the SJ-4 satellite to satisfy scientific requirements.

- Manufactures the SJ-4 satellite on schedule

- Integrates the scientific payloads on board the satellite

- Integrates the satellite on board the rocket

- Launches the SJ-4 satellite to the correct orbit.

A joint team of experts from these institutes was organized to co-ordinate the plan and schedule, and the collaboration was working well.

There were five scientific payloads, including the electrostatic analyzer and high energy particle telescope. The electrostatic analyzer measures heat plasma, such as that in the region of the earth stationary orbit ejected from the magnetospheric tail and that in the near-earth space penetrated from the magnetospheric plasma. The electrons and protons may be monitored in the energy range $0.1-40 \mathrm{keV}$ by 16 energy channels with an energy resolution of 15-20\%. The high energy particle telescope consisted of a semiconductor monitor. Two sensors were used to cover the whole range of the projecting angle distribution. The main properties of the telescope were given in Table 4 . The data will be analyzed 
Table 4. Properties of the high energy particle telescope

\begin{tabular}{lccc}
\hline Particle & Electron & Proton & Heavy nucleus \\
\hline -Energy range & $0.5-4.0 \mathrm{MeV}$ & $4.0-300 \mathrm{MeV}$ & $4.0-25 \mathrm{MeV}$ \\
$\begin{array}{l}\text { Energy channel } \\
\text { Half expansive } \\
\text { angle }\end{array}$ & 5 & 8 & 1 \\
\hline
\end{tabular}

together with those obtained at the cosmic ray stations on the ground in China.

The project of the SJ-4 scientific satellite is the second step of the small scientific satellite project in China, and we expect to arrange the next step in the future.

\section{PROSPECTS AND CONCLUSIONS}

Space science is usually considered as an important part of national space activity, and should be developed co-ordinately with space technology and space application. After a long period of development of space technology in China, the basis for the development of space science is now available. There have been space scientific experiments every year on board the Chinese satellite since 1987, and the scientific satellite programs have also been started in the last few years. It could be expected that the scientific satellite program will be arranged step by step depending upon the scientific requirements and opportunity. The large scientific mission will be arranged from a long term view point, however, the missions will be small in China at the present time and in the near future.

Space science is an important and front field of natural science, the space experiments and measurements are the basis for space science. The Chinese Academy of Sciences will pay more attention to the development of space science in the future, and we hope to arrange more space scientific activities. However, CAS can only carry out space activities at a level which depends on the financial support from the national programs. It seems that the situation has improved gradually for space science research, and we maintain our optimism for the future. As the scientist and user, the experts of CAS are preparing several proposals for scientific satellites, and hope that a national plan for the scientific satellite can be arranged in the future. However, from the view point of reality, the project of a scientific satellite can only be arranged in step by step for the present period.

An important experience for developing the Chinese scientific satellite is the interagency collaboration. Space technology has been developed by MAS in the last 30 years. Thus it is not necessary to organize another system for satellite and rocket technology, and transferring the developed space technology from MAS to the scientific satellite is an effective and low-cost approach. Therefore, the scientists and institutes of CAS will continue their collaboration with the experts and institutes of MAS.

The purpose of space science is to understand the natural rules, which belong and benefit all human beings. As a developing country, China has only a limited capability in the field of space science. Chinese scientists would like to do their best to contribute to and promote the co-operation between the international community of space science, and also of the small scientific satellite.

\section{REFERENCES}

I. W. R. Hu, D. R. Lu and Z. S. Lu. Space scientific experiments in China. 28th Plenary Meeting of $C O S P A R, 25$ June 6 July, The Hague, The Netherlands (1990).

2. S. C. Tu. An overview of the Chinese Space Program. In Der Weltraum Seit 1945 (Edited by Spillmann K. R.), p. 159. Birkhauser, Berlin (1987).

3. W. R. Hu, B. R. Zhang and D. R. Lu. Advances in Solar-Terrestrial Sciences of China. Science Press, Beijing (1992)

4. W. R. Hu, B. R. Zhang and D. R. Lu. The program of global character research of solar-terrestrial system in 22nd solar cycle in China. Adv. Space Res. 11, 57 (1991). 